\title{
Activation Analysis of Carbon and Nitrogen in Iron, Cobalt and Chromium
}

\author{
By M. Isshiki*, Y. Fukuda** and K. Igaki*
}

\begin{abstract}
Activation analyses of carbon and nitrogen in pure iron, cobalt and chromium have been developed using nuclear reactions, ${ }^{12} \mathrm{C}(\gamma, \mathrm{n}){ }^{11} \mathrm{C}$ and ${ }^{14} \mathrm{~N}(\mathrm{p}, \alpha){ }^{11} \mathrm{C}$. Activated carbon was separated in the form of $\mathrm{CO}_{2}$ by fusing the specimen at $1573 \mathrm{~K}$ in oxygen atmosphere. It was found that the contents of carbon and nitrogen are reliably determined in the level of 50 and $10 \mathrm{ppb}$, respectively. The effect of the hydrogen treatment is also confirmed on the removal of carbon and nitrogen contained in iron, chromium and cobalt.
\end{abstract}

(Received December 27, 1985)

Keywords: activation analysis, carbon, nitrogen, cobalt, iron, chromium, hydrogen treatment, zirconium treatment

\section{Introduction}

For the investigation of the substantial properties of transition metals and the influence of trace impurities on them, a well defined specimen of high purity must be used, because physical and chemical properties of the transition metals are remarkably affected by the presence of tiny amounts of impurities, such as carbon, nitrogen and oxygen. On the other hand, from the aspect of the steel making, high purity steels with low concentrations of nonmetallic elements are desired. For these reasons, analytical method with lower detection limits should be developed.

Activation analyses by means of charged particles and gamma-ray are known to be the most sensitive analytical methods for non-metallic light elements and were examined in detail by Engelmann $^{(1)(2)}$. There are, however, few papers on the activation analyses of light elements in transition metals. Blümel et al. ${ }^{(3)}$ reported the contents of carbon, oxygen and nitrogen in iron specimens annealed in an atmosphere of dry hydrogen, obtained by the

* Department of Materials Science, Faculty of Engineering, Tohoku University, Sendai 980, Japan.

** Graduate Student, Tohoku University, Present address: Research and Development Division, Victor Company of Japan, 12-3 Moriya-cho, Kanagawa-ku, Yokohama 221, Japan. charged particle activation analysis, without giving any detailed analytical procedures. Hislop et al. ${ }^{(4)}$ applied gamma-photon activation analysis to carbon in nickel. They fused the irradiated specimens in an oxygen atmosphere for the separation of the activated carbon.

The charged particle activation analysis of oxygen has been developed and reported by the present authors ${ }^{(5)}$. The present paper describes about activation analyses of carbon and nitrogen in iron, cobalt and chromium. Using the activation analyses developed, the effect of hydrogen treatment on the solid state purification has been examined. In the case of the chromium, zirconium treatment has been attempted by referring to the report by Hirano et al. ${ }^{(6)}$.

\section{Experimental Procedure}

\section{Specimens and treatment}

The cobalt specimens used were those purified by an anion exchange method and dry hydrogen treatment ${ }^{(7)}$ and the chromium specimens by floating zone-refining in dry hydrogen and hydrogen treatment at $1773 \mathrm{~K}^{(8)}$. Commercial high purity iron purchased from Johnson-Matthey Co. was used as the iron specimens.

For the annealing of the iron and cobalt 
specimens, Pd-purified hydrogen was used, and for the chromium specimens hydrogen purified by passing through a zeolite column and a liquid nitrogen trap. A special grade high purity alumina tube with the nominal purity of 4 nines was used for the high temperature hydrogen treatment of the chromium specimens. The temperatures during hydrogen treatment were kept at 1073, 1123 and $1773 \mathrm{~K}$ for iron, cobalt and chromium, respectively. The thickness of all the disk shape specimens for the heat treatment was $0.5 \mathrm{~mm}$.

In the case of the zirconium treatment, chromium specimens were wrapped in zirconium foils $20 \mu \mathrm{m}$ in thickness, annealed in evacuated quartz ampoules for $2 \mathrm{~h}$ at $1373 \mathrm{~K}$, and furnace-cooled. The surface contaminated in contact with zirconium was etched away by $\mathrm{HF}(10 \%)$, and then the specimens were polished electrochemically.

\section{Activation}

Nuclear reactions used for the analysis of $\mathrm{C}$ and $\mathrm{N}$ are ${ }^{12} \mathrm{C}(\gamma, \mathrm{n}){ }^{11} \mathrm{C}$ and ${ }^{14} \mathrm{~N}(\mathrm{p}, \alpha){ }^{11} \mathrm{C}$, respectively. The specimen for the analysis of carbon was vacuum sealed in a quartz tube with a flux monitor of iron and irradiated for $20 \mathrm{~min}$ with gamma-rays through the bremsstrahlung of 30 $\mathrm{MeV}$ electrons accelarated by LINAC in the Laboratory of Nuclear Science, Tohoku University. The weights of the specimens and the iron flux monitor were about 300 and 200 $\mathrm{mg}$, respectively. The current of the electron beam was $120-150 \mu \mathrm{A}$.

In the case of the nitrogen analysis, the specimen was mounted on a target holder and bombarded with $15 \mathrm{MeV}$ proton beam for 20 min through a collimeter $(5$ or $8 \mathrm{~mm}$ in diameter) and a flux monitor (Ti foil $20 \mu \mathrm{m}$ in thickness) in a helium atmosphere. The current of the proton beam was $1-2 \mu \mathrm{A}$. Since the cross section of $(p, n)$ reaction for ${ }^{52} \mathrm{Cr}$ is large, the current of proton beam for chromium specimen was diminished to $0.1 \mu \mathrm{A}$ to avoid suffering from superfluous bombing during handling of the activated specimens. The experiments were carried out in the Cyclotron Radioisotope Center, Tohoku University.

Special attention must be paid to the interference nuclear reactions which produce the same radionuclide as marked one. The interferences are estimated from Engelmann's results ${ }^{(1)(2)}$ to be negligibly small in the present experimental conditions.

\section{Analysis}

After bombardment, the surface was slightly etched away to remove the contaminated surface portion. The $\mathrm{Co}$ and $\mathrm{Cr}$ specimens were heated with appropriate amounts of tin and iron at $1573 \mathrm{~K}$ in a flow of oxygen to oxydize ${ }^{11} \mathrm{C}$ to $\mathrm{CO}_{2}$, and the evolved gas was absorbed in a dilute aqueous solution of $\mathrm{NaOH}$. The iron specimens were heated only with tin. The operation was finished about $30 \mathrm{~min}$ after the termination of the irradiation, in a comparable time to the half-life of ${ }^{11} \mathrm{C}$ of $20.38 \mathrm{~min}$. After the separation, the radioactivity of ${ }^{11} \mathrm{C}$ was measured by counting gamma-rays of $511 \mathrm{keV}$ emitted through positron annihilation using a $\mathrm{Ge}(\mathrm{Li})$ detector and a $4096 \mathrm{ch}$. PHA. The carbon and nitrogen contents were determined through the comparison of radioactivity from the specimens with that from the reference iron specimen of known contents. Details of the charged particle activation analysis were described in a previous paper ${ }^{(5)}$.

\section{Results and Discussion}

\section{Activation and separation}

Gamma-ray spectra measured on the asirradiated cobalt specimen and the $\mathrm{NaOH}$ solution containing ${ }^{11} \mathrm{C}$ from the same specimen are shown in Fig. 1. As seen from the figure, ${ }^{58} \mathrm{Co}$ is produced from stable isotope of cobalt, ${ }^{59} \mathrm{Co}$, through the reaction $(\gamma, \mathrm{n})$. Since ${ }^{58} \mathrm{Co}$ decays through positron emission. A511 includes the gamma-rays from ${ }^{58} \mathrm{Co}$ in addition to those from ${ }^{11} \mathrm{C}$. For this reason, the separation of ${ }^{11} \mathrm{C}$ is requisite for detecting a trace amount of carbon. Figure 1(b) indicates that ${ }^{11} \mathrm{C}$ is clearly separated from the irradiated cobalt specimen. Similar results were obtained for iron and chromium. Figure 2 shows decay curves of A511 keV gamma-ray peaks measured on the $\mathrm{NaOH}$ solutions containing ${ }^{11} \mathrm{C}$ from the cobalt specimen and the iron used as the reference specimen. Their half-lives 


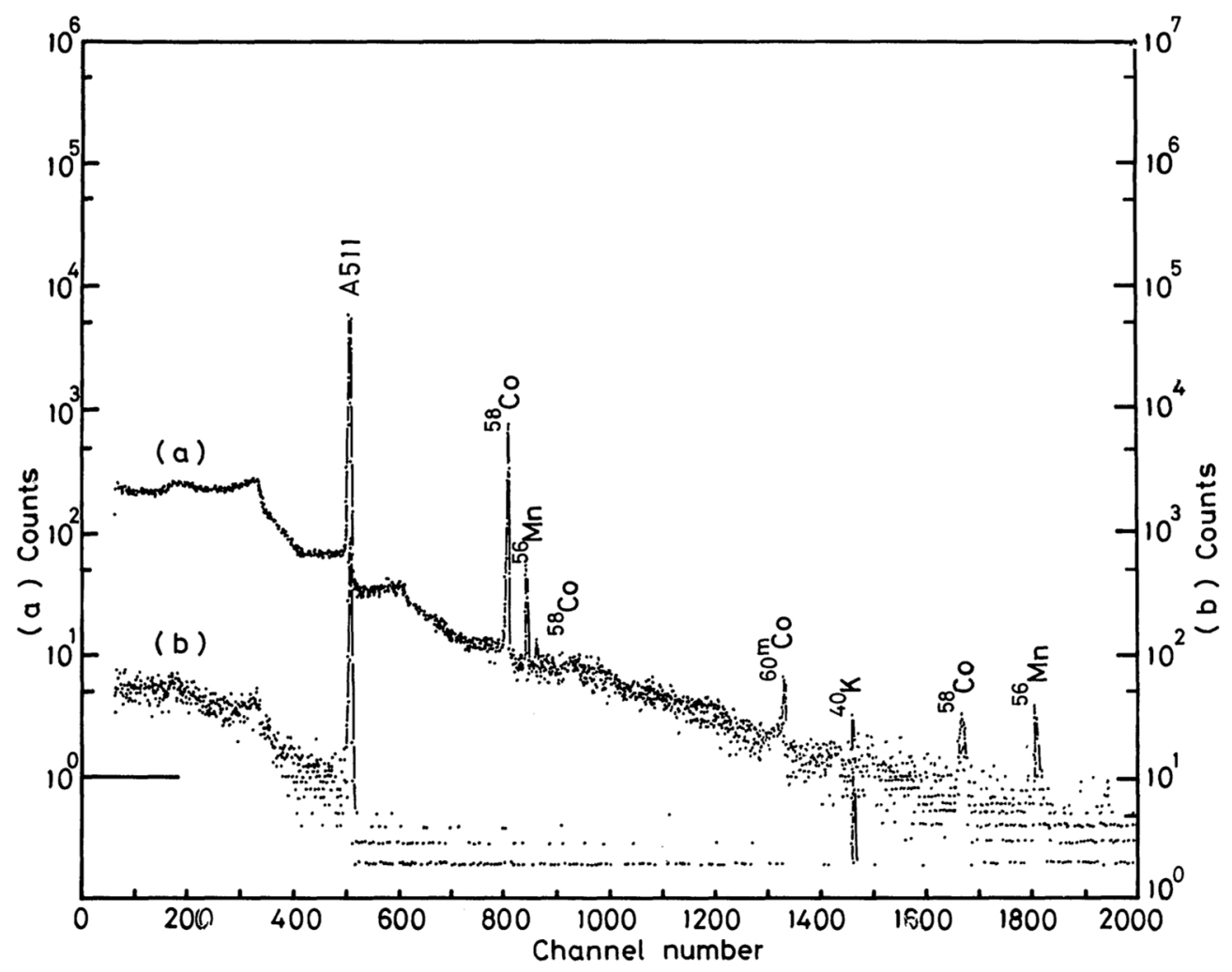

Fig. 1 Gamma-ray spectra measured on cobalt specimen irradiated (a) and the $\mathrm{NaOH}$ solution containing ${ }^{11} \mathrm{C}$ (b). A511 indicates the gamma-ray peak through positron annihilation on the decay of ${ }^{11} \mathrm{C}$.

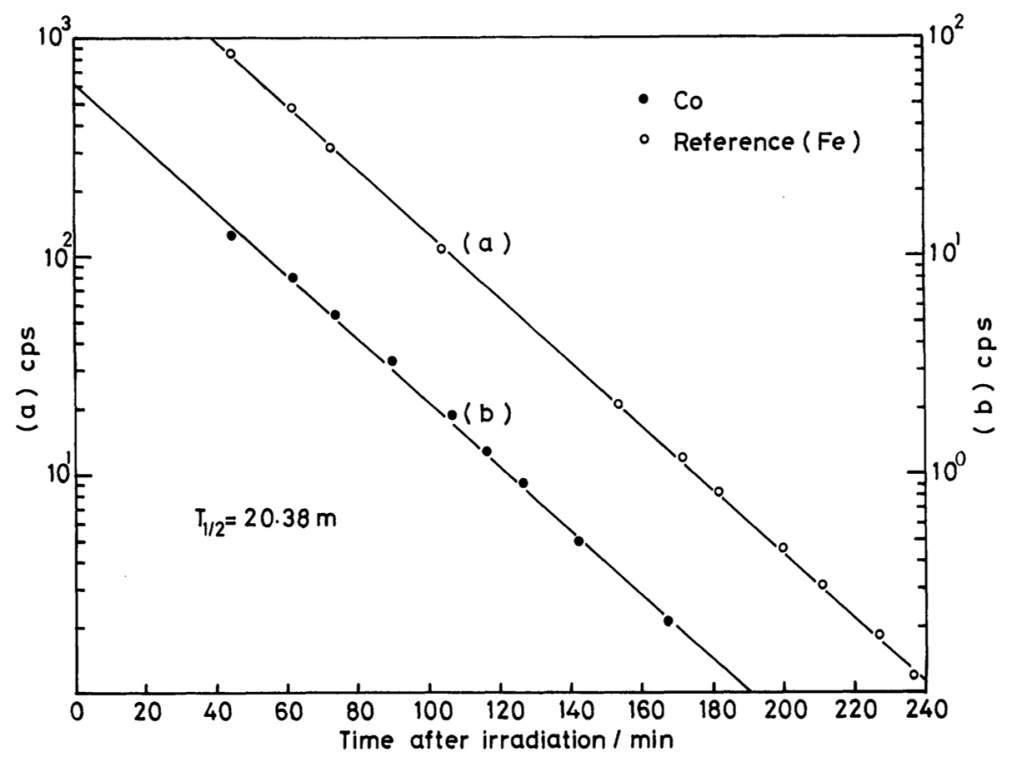

Fig. 2 Decay curves of A511 gamma-ray peaks of cobalt specimen and the iron used for the reference sample with the known carbon content. 
agree with $20.38 \mathrm{~min}$ of ${ }^{11} \mathrm{C}$, and this also shows that ${ }^{11} \mathrm{C}$ is effectively separated by the method adopted in the present paper. Judging from the levels of gamma-ray counting rate, carbon contents and the background level of circumstance, the detection limit is estimated at about $50 \mathrm{ppb}$ in the present experimental conditions.

In the case of nitrogen analysis, ${ }^{11} \mathrm{C}$ was separated in the same manner as the carbon analysis. Decay curves of A511 gamma-ray peaks of the iron specimens having known nitrogen contents reveal that the detection limit of nitrogen is estimated at $10 \mathrm{ppb}$. Since the average current to the chromium target was diminished because of its high yield of $(p, n)$ reaction, the values of the detection limits in chromium increased by about one order.

\section{Analysis}

Analytical results on iron containing carbon of $10-100 \mathrm{ppm}$ agree with those obtained by a conventional coulometric method within $5 \%$. This means that the activation analysis developed in the present paper show a good reproducibility.

The carbon and nitrogen contents in cobalt, chromium and iron specimens are summarized in Table 1. It is clearly observed that the hydrogen treatment is effective to remove carbon and nitrogen.

It is found that the $\mathrm{Zr}$ treatment is effective for the elimination of nitrogen in chromium as reported by Hirano et al. ${ }^{(7)}$. They found, in their measurements of the residual resistivity and internal friction, that the $\mathrm{Zr}$ treatment

Table 1 Analysis of $\mathrm{C}$ and $\mathrm{N}$ in the specimens treated in various manners.

\begin{tabular}{lccc}
\hline \multirow{2}{*}{ Specimen } & Treatment & \multicolumn{2}{c}{$\begin{array}{c}\text { Concentration } \\
\text { (mass ppm) }\end{array}$} \\
\cline { 3 - 4 } & & Carbon & Nitrogen \\
\hline \multirow{2}{*}{ Chromium } & Starting material & 21 & 287 \\
& Hydrogen treated & 4.9 & - \\
& Zr-treated & 20 & 85 \\
\hline \multirow{2}{*}{ Cobalt } & Annealed in vacuum & 22 & 16 \\
& Hydrogen treated & 1.7 & 1.5 \\
\hline \multirow{2}{*}{ Iron } & as-rolled & 43 & 4.2 \\
& Hydrogen treated & 0.8 & 1.3 \\
\hline \hline
\end{tabular}

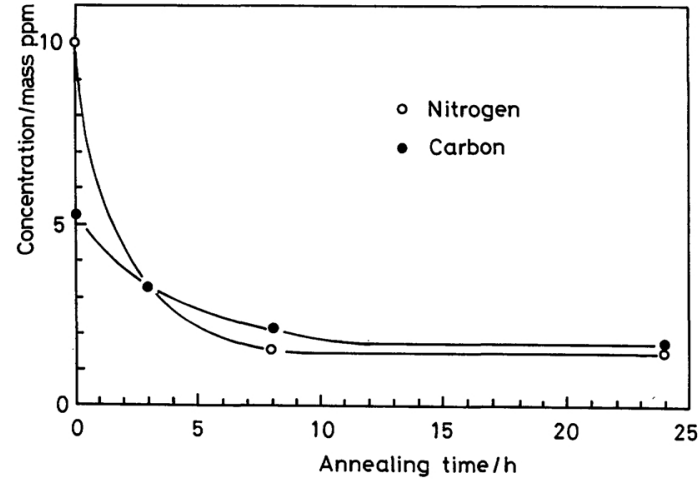

Fig. 3 Time dependences of the carbon and nitrogen contents of the cobalt specimen annealed in dry hydrogen at $1123 \mathrm{~K}$.

decreased the nitrogen concentration in chromium. It is also known that the $\mathrm{Zr}$ treatment hardly removes carbon in chromium. Experiment on the activation analysis of oxygen shows that the $\mathrm{Zr}$ treatment is available for the removal of oxygen.

Figure 3 shows the time dependence of the carbon and nitrogen contents of the cobalt specimens annealed in dry hydrogen at $1173 \mathrm{~K}$. These values are found to decrease exponentially with the increase of the annealing time. Theoretical analysis of the carbon concentration reveales that the decarburization process of cobalt under the present experimental conditions is rate-controlled by a surface reaction. Details of these results are reported elsewhere ${ }^{(7)}$.

\section{Conclusion}

Activation analyses of carbon and nitrogen in cobalt, iron and chromium have been attempted. Detection limits of carbon and nitrogen are estimated at 50 and $10 \mathrm{ppb}$, respectively. These methods would be easily applied to other metals and alloys in which the contained carbon can be separated by the conventional fusion method. The effects of hydrogen and zirconium treatments have been clarified.

\section{Acknowledgements}

The authors wish to express their gratitudes to the members of the Cyclotron Radioisotope Center and the Laboratory of Nuclear Science, Tohoku University. They also thank Mr. S. 
Fukutake, Mr. T. Shibata, Mr. K. Chiba, Mr.

K. Arakawa and Dr. M. Nagano for their help on performing the experiments.

\section{REFERENCES}

(1) C. Engelmann: J. Radioanal. Chem., 7 (1971), 281.

(2) C. Engelmann: J. Radioanal. Chem., 6 (1970), 399.

(3) G. Blümel, C. Engelmann and P. Reimers: Phys. Stat. Sol (a), 10 (1972), k69.

(4) J. S. Hislop, T. W. Sanders, T. J. Weber and D. R.
Williams: AERE-R-8182 (1975).

(5) M. Isshiki, K. Arakawa, S. Satoh, M. Nagano, Y. Fukuda, K. Chiba and K. Igaki: Trans. Iron Steel Inst. Japan, 23 (1983), 796.

(6) S. Hirano, O. Yoshinari and M. Koiwa: J. Less-Common Metals, 113 (1985), 17.

(7) M. Isshiki, Y. Fukuda and K. Igaki: J. Less-Common Metals, 105 (1985), 211.

(8) M. Isshiki, K. Arakawa, K. Igaki, A. Mizohata and T. Tsujimoto: J. Less-Common Metals, 96 (1984), 157. 